\title{
Jaundice Associated with Pseudomonas aeruginosa Bacteremia Complicating Acute Leukemia
}

\author{
Hisashi Funada, Tamotsu Matsuda and Yoshikatsu OKadA*
}

\begin{abstract}
Two adult patients with acute leukemia developed marked jaundice during Pseudomonas aeruginosa bacteremia. The progressive increase in serum conjugated bilirubin levels was disproportionate to the gradual decrease in serum alkaline phosphatase and transaminase activity to or below low normal. The production of coagulation factors decreased. Autopsy revealed periportal cholestasis with minimal liver-cell damage. These findings suggested decreased metabolic activity of liver cells associated with bacteremia, probably leading to impaired bilirubin excretion. Both patients died despite appropriate antibiotic therapy. Isolated hyperbilirubinemia, thus, seemed to be an ominous prognostic sign in severe infection.
\end{abstract}

(Internal Medicine 34: 100-103, 1995)

Key words: conjugated hyperbilirubinemia, intrahepatic cholestasis

\section{Introduction}

Clinical jaundice is a relatively uncommon complication of bacteremia in adults $(1,2)$. We describe here two patients with acute leukemia who developed marked conjugated hyperbilirubinemia during Pseudomonas aeruginosa bacteremia, with no evidence of extrahepatic biliary obstruction or significant hepatic parenchymal damage. The decreased metabolic activity of liver cells associated with bacteremia seemed to play a role in the pathogenesis of jaundice in our patients.

\section{Patients Studied}

During the period of 1972-1991,56 episodes of $P$. aeruginosa bacteremia occurred in 53 patients with acute leukemia in our department. Eight patients (15\%) developed clinical jaundice in the course of bacteremia. Six of them had a preexisting clinical hepatobiliary disease or a concurrent systemic cytomegalovirus infection, or had recently received potentially hepatotoxic medications. Thus, only two patients (4\%) formed the basis of this study. Their clinical and autopsy records are reviewed.

\section{Case Reports}

\section{Patient 1}

A 75-year-old man presented with fever and leukocytosis, and a diagnosis of acute myelogenous leukemia was made. He underwent antileukemic therapy with cytarabine and etoposide while empirically placed on cefmetazole and gentamicin. Fever continued, however, and he complained of abdominal pain, bloody diarrhea, and anal pain on bowel movements. Ecthyma gangrenosum of the anoperineal region appeared. He became jaundiced. Hepatitis B surface antigen ( $\mathrm{HBsAg}$ ) was not detected. Blood cultures grew Pseudomonas aeruginosa, and the same organism was recovered from stool. Ultrasonography revealed no significant hepatobiliary abnormalities except for gallstones. Antibiotic therapy was changed to piperacillin and gentamicin, both of which were in vitro active against the blood isolate. His granulocyte counts, however, remained below 500/ $\mu \mathrm{l}$ during therapy. The jaundice deepened, and he died two weeks after the onset of bacteremia.

Autopsy revealed bile plugs within bile ductules particularly at the edges of the portal tracts, with little or no injury to the liver cells (Fig. 1). Leukemic cell infiltration was slight in the portal tracts. No evidence of large bile-duct obstruction was found despite the presence of gallbladder stones. In addition, necrotizing enterocolitis with multiple ulcerations was observed particularly in the ileum, colon, and rectum, extending to the anoperineal ecthymatous lesion.

\section{Patient 2}

A 72-year-old woman was referred for evaluation and treatment of acute myelogenous leukemia accompanied by dissemi-

From the Protected Environment Unit and the Third Department of Medicine and *the First Department of Pathology, Kanazawa University School of Medicine, Kanazawa

Received for publication May 9, 1994; Accepted for publication September 26, 1994

Reprint requests should be addressed to Dr. Hisashi Funada, the Protected Environment Unit and the Third Department of Medicine, Kanazawa University School of Medicine, Kanazawa 920 
nated intravascular coagulation (DIC). On admission she had a low-grade fever, and cefmetazole and gentamicin were started

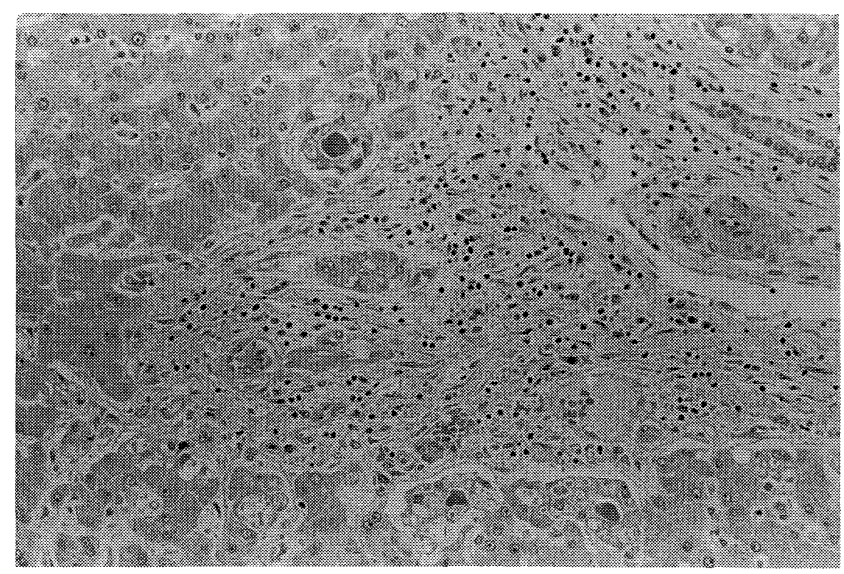

Fig. 1. Section of liver shows inspissated bile plugs within bile ductules at the edges of the portal tract, with minimal parenchymal damage $(\mathrm{HE}, \times 160)$. on an empirical basis. Antileukemic therapy with daunorubicin, enocitabine, neocarzinostatin, and prednisolone was given in combination with argatroban, a synthetic thrombin inhibitor. DIC resolved concomitantly with a rapid decrease in the leukemic cell count. Despite such anticoagulant therapy, however, left hemiparesis occurred, and an indwelling urinary catheter was inserted. Fever tended to increase. Blood cultures grew Pneudomonas aeruginosa, the same organism being recovered from urine. Soon thereafter she became jaundiced. HBsAg was not detected. Ultrasonography disclosed no identifiable abnormalities in the liver. Antibiotic therapy was changed to imipenem/cilastatin and amikacin, to which the isolate was sensitive. However, the jaundice deepened. Two weeks later her clinical course was complicated by another bacteremia due to Enterobacter cloacae. She died in septic shock, with no evidence of marrow recovery.

At autopsy, an extensive cerebral infarct was seen in the right hemisphere. Mild bile retention with some plugging of ductules was observed, chiefly at the periphery of the portal tracts, with minimal or no evidence of parenchymal damage. However,
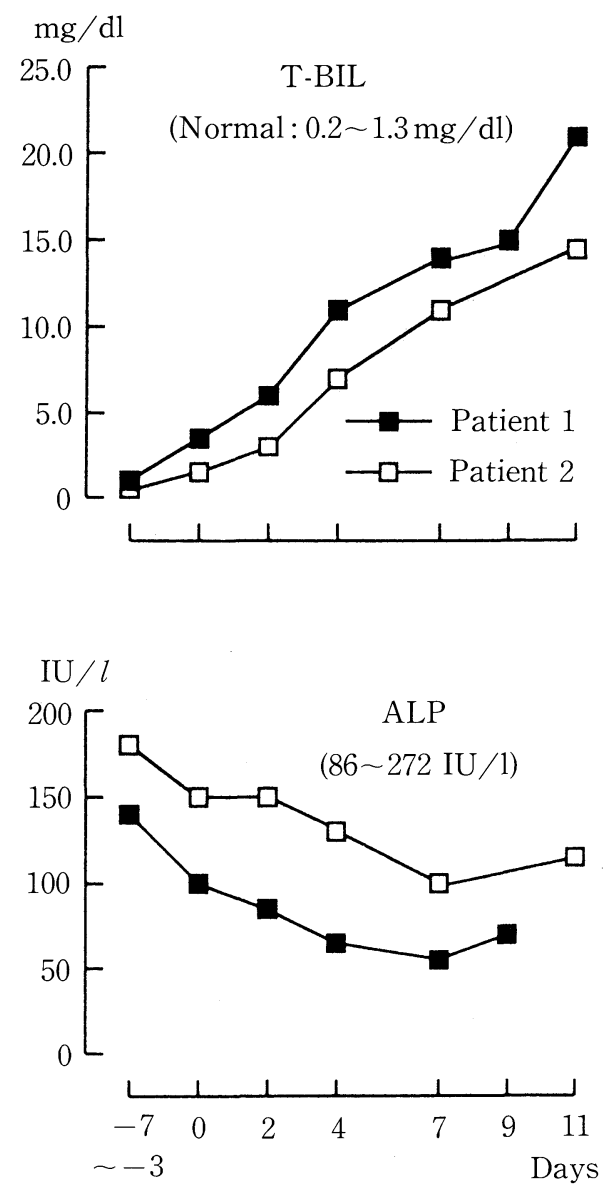

Day 0: day of onset of bacteremia
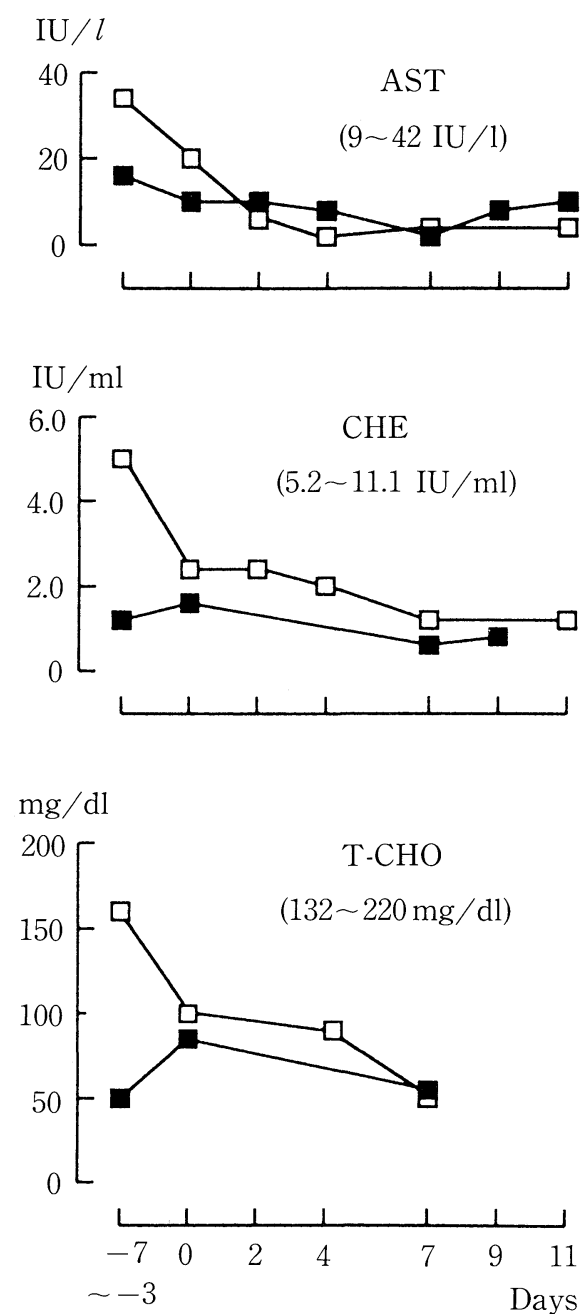

Fig. 2. Serial liver function studies in patients with Pseudomonas aeruginosa bacteremia and jaundice. T-BIL: total bilirubin, AST: aspartate aminotransferase, ALP: alkaline phosphatase, CHE: choline esterase, T-CHO: total cholesterol. 
there was no demonstrable hepatobiliary disease, although mild leukemic cell infiltration was noted in the portal tracts, but there was no bile duct obstruction.

\section{Changes in Liver Function Tests}

The serial liver function studies in two patients are shown in Fig. 2. Conjugated bilirubin represented $71 \%$ of the concurrent peak level of total serum bilirubin (T-BIL) in Patient 1 and $72 \%$ in Patient 2. The activities of serum alkaline phosphatase (ALP), and aspartate aminotransferase (AST), which were initially within normal limits in both patients, tended to fall gradually to or below low normal as the jaundice deepened. This was also the case with serum $\gamma$-glutamyl transpeptidase, leucine aminopeptidase, and alanine aminotransferase (ALT). Serum lactic dehydrogenase seemed to follow a similar course, but the activity depended largely on the leukemic process. On the other hand, serum choline esterase (CHE) and total cholesterol (T-CHO) in Patient 2 showed essentially the same pattern of changes as the other enzymes, whereas both levels in Patient 1 remained below low normal. Serum albumin level exceeded $3.0 \mathrm{~g} / \mathrm{dl}$ by replacement treatment in Patient 1 , while such a treatment proved unsuccessful in Patient 2.

Blood coagulation studies performed after the development of jaundice revealed prolongation of the prothrombin time and activated partial thromboplastin time, and low levels of fibrinogen and antithrombin III in both patients (Table 1). A low level of factor $\mathrm{V}$ was also observed in Patient 1. Fibrin degradation products were not increased in either patient, with the prothrombin time remaining almost unchanged despite intravenous vitamin K therapy. Such hemostatic defects were thus interpreted as evidence of decreased synthesis of coagulation factors in the liver.

Patient 1 displayed virtually the same pattern of hemostatic

Table 1. Blood Coagulation Studies before and after the Onset of Pseudomonas Aeruginosa Bacteremia

\begin{tabular}{|c|c|c|c|c|}
\hline \multirow[b]{2}{*}{ Day of study ${ }^{b}$} & \multicolumn{2}{|c|}{ Patient 1} & \multicolumn{2}{|c|}{ Patient $2^{\mathrm{a}}$} \\
\hline & Day -5 & Day +11 & Day -5 & Day +11 \\
\hline Prothrombin time & \multicolumn{2}{|c|}{$(12.9 \pm 1.0 \mathrm{sec})^{\mathrm{c}}$} & \multicolumn{2}{|c|}{$(11.9 \pm 1.0 \mathrm{sec})$} \\
\hline $\begin{array}{l}\text { Activated partial } \\
\text { thromboplastin time }\end{array}$ & ${ }_{(<}^{64.0}$ & $\begin{array}{l}62.2 \\
\mathrm{sec})\end{array}$ & 61.9 & $\begin{array}{l}45.1 \\
\mathrm{sec})\end{array}$ \\
\hline Fibrinogen & $\begin{array}{l}132 \\
(170\end{array}$ & $\begin{array}{r}138 \\
\mathrm{mg} / \mathrm{dl})\end{array}$ & $\begin{array}{l}130 \\
(170\end{array}$ & $\begin{array}{r}173 \\
0 \mathrm{mg} / \mathrm{dl})\end{array}$ \\
\hline $\begin{array}{l}\text { Fibrin degradation } \\
\text { products }\end{array}$ & $\begin{array}{r}5-<10 \\
\quad(<1\end{array}$ & $\begin{array}{c}5-<10 \\
\lg / \mathrm{ml})\end{array}$ & \multirow{2}{*}{\multicolumn{2}{|c|}{$(<5 \mu \mathrm{g} / \mathrm{ml})$}} \\
\hline \multicolumn{3}{|c|}{$(100 \pm 30 \%)$} & & \\
\hline Antithrombin III & $(30 \pm 5 \mathrm{mg} / \mathrm{dl})$ & $\begin{array}{c}7.9 \\
\mathrm{mg} / \mathrm{dl})\end{array}$ & $\begin{array}{r}83.6 \\
\quad(10\end{array}$ & $\begin{array}{l}37.5 \\
30 \%)\end{array}$ \\
\hline
\end{tabular}

${ }^{\text {a }}$ DIC secondary to leukemia per se had resolved by the time Pseudomonas aeruginosa bacteremia occurred.

${ }^{b}$ The day of onset of bacteremia was designated as day 0 .

${ }^{c}$ Figures in parentheses indicate normal ranges. defects prior to and after the onset of jaundice. On the other hand, Patient 2 developed DIC secondary to her leukemia, but the DIC had resolved by the time Pseudomonas aeruginosa bacteremia and jaundice occurred.

\section{Discussion}

Clinical jaundice seems to occur more frequently among patients with $P$. aeruginosa bacteremia than in those with other forms of gram-negative sepsis $(1,3,4)$. It previously occurred in $52 \%$ and $26 \%$ of the patients with Pseudomonas aeruginosa bacteremia studied by Forkner et al (3) and Flick and Cluff (4), respectively. The majority of their patients had neoplastic diseases, including hematologic malignancies and solid tumors. Twenty-two of the 23 patients reported by Forkner et al (3) died with a median survival of 4.0 days following the first positive blood culture. On the other hand, only two patients $(4 \%)$ in our acute leukemia series had jaundice concurrently with their bacteremia. No pathophysiologic mechanisms peculiar to $\mathrm{Pseu}$ domonas aeruginosa-associated jaundice have been elucidated yet (5). The reduced frequency, therefore, seems to be due largely to improved therapeutic management of both the underlying illness and associated infectious complications.

Jaundice has recently become recognized as a concomitant condition of bacteremia, irrespective of whether it is gramnegative or gram-positive, in adults (2, 6-8). Consistent biochemical features have been reported to include conjugated hyperbilirubinemia with disproportionately low levels of serum ALP, transaminases and T-CHO, despite histologic evidence of intrahepatic cholestasis (2, 6, 7). Miller et al (6) and later Franson et al (7) reported that these enzyme levels are elevated to a mean almost 2-fold the high normal level, with T$\mathrm{CHO}$ remaining normal. In both of the present patients, however, all the liver enzyme activities studied tended to begin and remain below low normal or decrease gradually to that level, despite the progressive deepening of jaundice. Such a marked decrease in liver enzyme activities was considered distinctive of the jaundice in these two patients. Moreover, the levels of coagulation factors, T-CHO, and serum albumin decreased or remained low, reflecting decreased hepatic reserve capacity.

Intrahepatic cholestasis, particularly in periportal bile ductules, with little or no liver cell injury, such as that seen in our patients, seems to be pathognomonic of jaundice in severe infection $(2,9)$. Neither of the patients had recently received potentially cholestatic therapeutic agents. Moreover, hemolytic processes, resolving hematomas, shock liver, acute viral hepatitis, pyogenic hepatic infection, and obstructive jaundice due to leukemic cell infiltration or gallstones were believed unlikely based on autopsy studies, as well as on available clinical and laboratory information. Incidentally, the serologic tests to establish a diagnosis of hepatitis $C$ were not yet routinely available when these patients were admitted.

Hyperbilirubinemia has been reported to occur as a consequence of severe infection $(2,6-9)$. The exact mechanism remains unknown, although decreased hepatic bilirubin clearance has been inferred to be the probable cause (2). In the 
present patients, the pattern of liver-function and coagulation abnormalities, along with the liver histology at autopsy, suggested decreased metabolic activity of liver cells in association with severe infection, which may have contributed to the impaired bilirubin excretion. It has recently been suggested that bacterial cell-wall components such as endotoxin and teichoic acid may adversely affect the hepatic excretory function (10, 11). Furthermore, there is growing evidence that such microbial products can cause organ dysfunction through the activation of humoral pathways as well as through the amplification of cytokine-mediated inflammatory responses (12).

Hemostatic deficiencies were seen prior to the onset of bacteremia in Patient 1 . This may have reflected the severity of his local infection. The decrease in coagulation factors synthesized in the liver may, thus, be a sensitive indicator portending jaundice in severe infection.

In the preantibiotic era, jaundice in severe infection indicated a poor prognosis (2). This seems to be the case even at present (8). Therefore, clinical jaundice may suggest that the infection is beyond control.

\section{References}

1) Vermillon SE, Gregg JA, Baggenstoss AH, Bartholomew LG. Jaundice associated with bacteremia. Arch Intern Med 124: 611, 1969.

2) Zimmerman HJ, Fang M, Utili R, et al. Jaundice due to bacterial infection. Gastroenterology 77: 362, 1979.

3) Forkner CE Jr, Frei E III, Edgcomb JH, Utz JP. Pseudomonas septicemia Observations on twenty-three cases. Am J Med 25: 877, 1958.

4) Flick MR, Cluff LE. Pseudomonas bacteremia. Review of 108 cases. Am J Med 60: 501, 1976.

5) Pollack M. Pseudomonas aeruginosa. in: Principles and Practice of Infectious Diseases, 3rd ed, Mandell GL, Douglas RG Jr, Bennett JE, Eds. Churchill Livingstone, New York, 1990, p.1673.

6) Miller DJ, Keeton GR, Webber BL, Saunders SJ. Jaundice in severe bacterial infection. Gastroenterology 71: 94, 1976.

7) Franson TR, Hierholzer WJ Jr, LaBrecque DR. Frequency and characteristics of hyperbilirubinemia associated with bacteremia. Rev Infect Dis 7: $1,1985$.

8) Watanakunakorn C, Chan SJ, Demarco DG, Palmer JA. Staphylococcus aureus bacteremia: significance of hyperbilirubinemia. Scand J Infect Dis 19: $195,1987$.

9) Lefkowitch JH. Bile ductular cholestasis: an ominous histopathologic sign related to sepsis and "cholangitis lenta." Hum Pathol 13: 19, 1982.

10) Utili R, Abernathy $\mathrm{CO}, \mathrm{Zimmerman} \mathrm{HJ}$. Inhibition of $\mathrm{Na}^{+}, \mathrm{K}^{+}$-adenosine triphosphatase by endotoxin: a possible mechanism for endotoxin-induced cholestasis. J Infect Dis 136: 583, 1977.

11) Rose HD, Lentino JR, Mavrelis PG, Rytel MW. Jaundice associated with nonhepatic Staphylococcus aureus infection: does teichoic acid have a role in pathogenesis? Dig Dis Sci 27: 1046, 1982.

12) Bone RC. The pathogenesis of sepsis. Ann Intern Med 115: 457, 1991. 